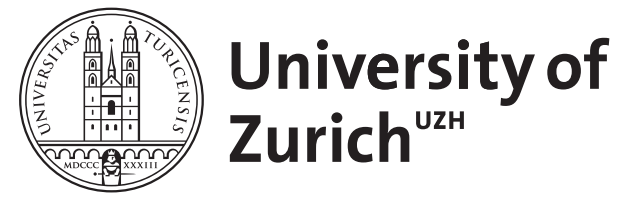

Zurich Open Repository and Archive

University of Zurich

University Library

Strickhofstrasse 39

CH-8057 Zurich

www.zora.uzh.ch

Year: 2012

\title{
Neue Drogen
}

Kupferschmidt, Hugo

DOI: https://doi.org/10.1024/1661-8157/a000923

Posted at the Zurich Open Repository and Archive, University of Zurich ZORA URL: https://doi.org/10.5167/uzh-71674

Journal Article

Accepted Version

Originally published at:

Kupferschmidt, Hugo (2012). Neue Drogen. Praxis, 101:565-570.

DOI: https://doi.org/10.1024/1661-8157/a000923 
DOI 10.1024/1661-8157/a000923

((PX 9_Rubrik CME))

\title{
Neue Drogen
}

Im Artikel verwendete Abkürzungen:

\author{
BZP 1-Benzylpiperazin \\ DOB 2,5-Dimethoxy-4-Bromoamphetamin \\ DOM 2,5-Dimethoxy-4-methamphetamin \\ LSD Lysergsäurediethylamid \\ mCPP m-Chlorophenylpiperazin \\ MDMA 3,4-Methylendioxy-N-methylamphetamin \\ MDPV Methylendioxypyrovaleron \\ MeOpp Paramethoxyphenylpiperazin \\ TFMPP Trifluoromethylphenylpiperazin
}

In den letzten wenigen Jahren sind auf dem Markt eine Vielzahl von neuen Designerdrogen aufgetaucht. Die Prävalenz des Gebrauchs dieser Substanzen ist vor allem in England und Skandinavien hoch. In der Schweiz wurden bisher erst vereinzelte Fälle bekannt, die aber vermutlich nur die Spitze des Eisberges darstellen. Ein Grund für diese geographischen Unterschiede liegt sicher in der unterschiedlichen Verfügbarkeit anderer (bisheriger) Drogen und in der Gesetzgebung, die ein wichtiger Treiber für die Entwicklung neuer Substanzen ist. Zudem erleichtert der Vertrieb über das Internet die Beschaffung dieser Rauschgifte. Für viele dieser neuen Drogen haben sich Bezeichnungen wie «Legal Highs» oder «Research Chemicals» eingebürgert, was ihren rechtlichen Status unterstreichen soll und den Bezug zum Missbrauch verschleiert. Die Vertreter dieser Drogengruppe haben mannigfaltige Strassenrufnamen, was zu Verwechslungen führen kann. Zudem werden sie unter irreführenden Verwendungszwecken angepriesen (Pflanzendünger, Badesalz, u.ä.). Bei den Kräutermischungen, die unter Bezeichnungen wie «Spice» und «K2» angepriesen werden, wurde zunächst (erfolglos) versucht, die berichteten Wirkungen den enthaltenen Pflanzenbestandteilen zuzuschreiben; im Jahr 2008 gelang es dann aber, darin undeklarierte künstliche Substanzen mit cannabinomimetischer Wirkung nachzuweisen (z.B. Aminoalkylindol JWH018).

Es lohnt sich, hier einen Überblick über diese Substanzgruppen zu geben, weil einerseits die klinische Wirkung der verschiedenen Stoffe viele Gemeinsamkeiten haben, und andererseits nie sicher ist, welche Substanz in den Produkten angetroffen wird, da deren Zusammensetzung immer wieder wechselt. Pilz- und Pflanzendrogen, die chemisch und wirkungsmässig mit den neuen Drogen verwandt sind, werden hier ebenfalls erwähnt.

Die Substanzen haben strukturelle Ähnlichkeit zu endogenen Neurotransmittern wie Serotonin, Noradrenalin und Dopamin und sind chemisch Abkömmlinge oder Analoga von Phenylethylamin (Kathinone, Amphetamine und andere), Piperazin, Piperidin, Phencyclidin und Tryptamin. Die Leitsubstanz Cathinon, die in der Pflanzendroge Khat (Cathaedulis) vorkommt, ist das Beta-Keto-Analog des Amphetamins («bk-Amphetamin»). Für die Methylendioxy-Varianten des Amphetamins (z.B. Ecstasy, MDMA) gibt es praktisch alle Entsprechungen bei den Cathinonen. Zu den Cathinonen gehören Methcathinon, Dimethylcathinon, Ethcathinon, Mephedron, Methedron, Pyrovaleron, die fluorierten Varianten 3-Fluoromethcathinon und 4-Fluoromethcathinon (Flephedron), sowie die Ethylendioxy-Varianten Butylon, Ethylon und Methylendioxypyrovaleron (MDPV). Die übrigen Vertreter der Phenethylamine stehen bei den neueren Drogen nicht im Vordergrund und werden daher hier nicht berücksichtigt. Zur Gruppe der Piperazine gehören 1-Benzylpiperazin (BZP, «A2») und die Phenylpiperazine Trifluoromethylphenylpiperazin (TFMPP), m-Chlorophenylpiperazin (mCPP) und Paramethoxyphenylpiperazin (MeOPP). Bei den Pipe- 
ridinen zu erwähnen sind Pipadrol, Desoxypipadrol («Ivory Wave») und Diphenylprolinol, die mit dem Methylphenidat verwandt sind. In dieselbe Klasse wie Phencyclidin («Angel Dust») gehören das als dissoziatives Anästhetikum verwendete Ketamin und das Methoxetamin. Zur Gruppe der Tryptamine, die sich mit ihrer Indolringstruktur von der Aminosäure Tryptophan herleiten, zählen Psilocybin und Psilocin aus den halluzinogenen Pilzen (Psilocybe spp.), LSD (Lysergsäurediethylamid) sowie eine lange Reihe von Designerstoffen, die hier nicht erwähnt werden können.

Davon abzugrenzen sind die Cannabinoidrezeptor-Agonisten («synthetische Cannabinoide»), die unter der Bezeichnung «Spice» vermischt mit Kräutern als Räuchermischungen im Internet zum Kauf angeboten werden. Da diese Stoffe in grosser Variation synthetisiert und auch publiziert werden, sind in denselben «Spice»-Mischungen zu unterschiedlichen Zeitpunkten nicht immer dieselben Wirkstoffe enthalten (z.B. JWH-073, JWH-200, JWH-250, HU-210, CP47,497 und seine Homologe, u.a.). Die JWH-Stoffe (nach dem Chemiker John Woodward Huffman) wurden eigentlich zur Erforschung der Rezeptorinteraktionen im Endocannabinoidsystem hergestellt. Diese Substanzen haben ein Wirkungsprofil, das deutlich über das der natürlichen Cannabinoide hinausgeht.

$\mathrm{Zu}$ diesen rein synthetischen Drogen gesellen sich zentralnervös wirksame Pflanzendrogen (Salvia divinorum, Argyreia nervosa, Datura spp., Mitragyna speciosa) und halluzinogene Pilze (Zauberpilze, Magic Mushrooms, Psilocybe spp.), die aber weiter weniger populär sind als die oben genannten Stoffe. Als Kratom werden Zubereitungen der Pflanze Mitragyna speciosa bezeichnet. 
g

Die meisten dieser neuen Drogen sind Stimulanzien (wie Amphetamin), einige haben (wie LSD) vorwiegend halluzinogene oder (wie Ecstasy) entaktogene Wirkung. Viele vereinigen alle drei Eigenschaften in unterschiedlicher Ausprägung. Es ist schlecht untersucht, wie gefährlich der Konsum dieser neuen Stoffe ist. Dennoch weisen erste Fallberichte schwerer Nebenwirkungen und Todesfällen darauf hin, dass sie ein gewisses Risiko bergen.

Diese neuen Drogen wirken unterschiedlich stark auf die verschiedenen zentralnervösen Rezeptoren ein. Das führt dazu, dass die klinischen Wirkungen der Einzelsubstanzen sich grösstenteils gleichen, die einzelnen Wirkungsqualitäten aber idealerweise unterschiedlich stark betont sind. Es ist aber mit einer erheblichen interindividuellen Variabilität zu rechnen, und es ist daher unmöglich, vom Symptombild auf eine bestimmte Substanz zu schliessen. Die neueren Stimulanzien wie 1-Benzylpiperazin, Mephedron, Naphyron oder Diphenylprolinolinhibieren die Wiederaufnahme von Monoaminen (v.a. Dopamin und Noradrenalin) und führen daher in erster Linie zu einem sympathomimetischen Toxidrom. Bei den Substanzen mit entaktogener Wirkung, zu denen die Phenylpiperazine oder das Methylon gehören, und Halluzinogene wie 5-Methoxy-N,N-Diisopropyltryptamin (5-MeODiPT) oder 2,5-Dimethoxy-4-Bromoamphetamin (DOB) ist das Wirkungsspektrum durch eine zentrale Serotoninausschüttung und/oder eine Hemmung des Serotonin-Reuptake geprägt. Die Halluzinogene wirken zudem als 5$\mathrm{HT}_{2}$-Agonisten. Serotoninerge Wirkungen stehen somit bei den entaktogenen und halluzinogenen Wirkstoffen im Vordergrund.

Neben den erwünschten Wirkungen, d.h. den stimulierenden, entaktogenen und halluzinatorischen Effekt mit einem Gefühl der Energie und Euphorie, erhöhter Aufmerksamkeit, verminderter Müdigkeit, Drosselung des Hungergefühls, Geschwätzigkeit und sexueller Erregbarkeit, treten stoffabhängig in unterschiedlichem Masse auch unerwünschte Wirkungen auf. Dazu gehören im Rahmen des sympathomimetischen Toxidroms die Unruhe bis Agitation, Palpitationen, Tachykardie, arterielle Hypertonie, Schwitzen und eine Mydriasis. Unangenehmer sind Schwindel, Dysphorie, Angst- und Spannungsgefühl, Kopfschmerzen (die migräneartig sein können), Schlaflosigkeit, Tremor, Trismus, Zähneknirschen, Myoklonien, extrapyramidale Störungen, Übelkeit, Erbrechen, Bauchschmerzen, Hyperventilation und Thoraxschmerzen. Zu den schwereren Nebenwirkungen sind Verwirrung, paranoide Psychose, schwere Aggression, die mit Selbstverstümmelung, Suizid und Gewalttaten begleitet sein können, metabolische Azidose, QT-Verlängerung und Krampfanfälle zu zählen. Bewusstseinsstörungen, Hyperthermie, Dehydratation, Hyponatriämie, Myokardinfarkte, Myokarditis, Kollaps und Atemversagen sind lebensbedrohliche Komplikationen. Todesfälle wurden beschrieben. DOB und 2,5-Dimethoxy-4-methamphetamin (DOM), aber auch die Benzodifurane («Bromodragonfly») können zu schweren anhaltenden arteriolären Vasokonstriktionen mit lebensbedrohlichen Ischämien führen. Vaskulitiden mit Hautnekrosen und Leukopenien können auch die Folge von des Anthelminthikums Levamisol sein, das dem Kokain in letzter Zeit als Streckmittel und Wirkungsverstärker beigemischt wird. Für einzelne Substanzen wurde die Entwicklung einer Abhängigkeit berichtet.

Das Wirkungsbild der synthetischen Cannabinoide vereinigt die Symptome, die von Tetrahydrocannabinol (dem Wirkstoff von Cannabis cativa ((Autor: meinen Sie Cannabis sativa?))) bekannt sind, mit denjenigen der Stimulanzien. Als unerwünschte Effekte werden injizierte Konjunktiven, Blässe, Hypertonie, Erbrechen, Tachykardie, Mundtrockenheit, Angstgefühle, Halluzinationen, Agitation, Zittern, Myoklonien und generalisierte Krampfanfälle beschrieben. Bei Ketamin und seinen Analogen Phencyclicin und Methoxetamin werden Euphorie, verzerrte Wahrnehmung und Halluzinationen gesucht, während Nausea, Erbrechen, Diarrhö, Paranoia und Angstgefühl als unerwünschte Wirkungen beschrieben werden.

Kratom wird wegen seines Gehalts an Mitragynin und anderer Alkaloide, die opiatähnliche Wirkungen haben, in asiatischen Ländern traditionell zum Opiatentzug und bei Husten verwendet. Mitragynin wirkt in niedrigen Dosen stimulierend, in höheren sedierend. 2011 wurde in Deutschland und in der Schweiz je ein Fall mit Kratomassoziierter Leberschädigung mitgeteilt. Die Aztekensalbei (Salvia divinorum) enthält Salvinorin A, das hochselek- 
tiv an den kappa-Opioidrezeptor bindet und so Halluzinationen, Diurese und eine Analgesie auf spinaler Ebene, aber keine Atemdepression verursacht. Diese Pflanzendroge wird gekaut oder geraucht; die enterale Resorption führt - wahrscheinlich durch einen First-pass-Effekt - nicht zu Halluzinationen. Unerwünschte Wirkungen sind Angstgefühl, Dysphorie, Verwirrung, Sprachstörungen, Kopfschmerzen und Schläfrigkeit. 
Abklärungsstrategie und Diagnostik ((Ü1))

Die Abklärung der Intoxikation mit all diesen Substanzen beruht weitgehend auf der Anamnese, der Analyse der Vergiftungsumstände und der Ausprägung der klinischen Symptome, wobei die Identifikation bestimmter Toxidrome (z.B. sympathomimetisches Syndrom, serotonerges Syndrom, halluzinatorisches Syndrom) hilfreich ist. Die Muttersubstanzen und ihre Metabolite sind zwar im Urin, teilweise auch im Blut nachweisbar, die entsprechenden Nachweise werden aber kaum im analytischen Routinebetrieb angeboten, sodass sie im klinischen Alltag nicht zur Verfügung stehen und allenfalls zur Diagnosesicherung aus forensischen oder wissenschaftlichen Gründen durchgeführt werden. Erschwerend kommt hinzu, dass sich die Substanzen in den auf dem Schwarzmarkt angebotenen Produkten zum Teil rasch abwechseln und damit jedes Mal breit gesucht werden muss.

Hohe Aufmerksamkeit verdient die Gefahr der Komplikationen. Daher ist es angezeigt, die Patienten klinisch und am Monitor zu überwachen, um eine Psychose, Hypertonie, Hyperthermie, Ischämie, eine Rhabdomyolyse oder einen Krampfanfall rechtzeitig zu diagnostizieren. Es soll mindestens ein 12-Ableitungs-EKG geschrieben werden. Labormässig sind Elektrolyte, Leber- und Muskelenzyme (inkl. Herzenzyme), der Säurebasenstatus und das Kreatinin zu messen.

Therapie/Prognose/Prävention ((Ü1))

Da für diese Drogen keine spezifischen Behandlungen und keine Antidote zur Verfügung stehen, ist die Therapie ausschliesslich supportiv bzw. symptomatisch. Bei Unruhe soll versucht werden, auf die Betroffenen in einer reizarmen Umgebung beruhigend einzuwirken («talk down»). Bei Angstzuständen, Agitation, Krampfanfällen und Hyperthermie ist eine Sedation mit Benzodiazepinen, in der Regel intravenös verabreicht, angezeigt. Auch eine supraventrikuläre Tachykardie bessert sich in der Regel damit. Kann eine Hyperthermie damit nicht innert kurzer Zeit in den Griff gebracht werden, oder liegt bereits eine lebensbedrohliche Temperaturerhöhung vor, muss zusätzlich mit physikalischen Mitteln gekühlt werden. Bei anhaltender therapiebedürftiger Hypertonie sind Vasodilatatoren (Nitrate, Nitroprussid) zu verwenden; Betablocker sind wegen der potenziellen Verschlimmerung der Hypertonie durch die ungebremste alphaadrenerge Stimulation kontraindiziert. Die Patienten sollen angemessen hydriert, die Elektrolytstörungen behoben werden.

Völlig asymptomatische Patienten können nach einigen Stunden entlassen werden, symptomatische Patienten nach dem Abklingen ihrer Symptome.

Sind die Symptome einmal abgeklungen, ist eine Restitutio ad integrum zu erwarten. Die Prognose und die Langzeitfolgen der akuten Vergiftung ist wesentlich vom Auftreten von Komplikationen abhängig. Über die Langzeitfolgen eines regelmässigen chronischen Konsums dieser neuen Substanzen ist nichts bekannt. Dauerschäden sind damit nicht völlig ausgeschlossen. Für einzelne Substanzen ist ein Abhängigkeitspotenzial beschrieben. 


\section{Fragen zu Neuen Drogen}

\section{Frage 1}

Die klinischen Wirkungen der zur Gruppe der Cathinone gehörenden Stoffe umfassen unter anderem folgende typische Symptome, ausser:

(Einfachauswahl, 1 richtige Antwort)
a) Tachykardie
b) Agitation
c) Erhöhung der Körpertemperatur
d) Vaskulitis
e) Arterielle Hypertonie

\section{Frage 2}

Nicht zur Gruppe der Cathinone gehört (Einfachauswahl, 1 richtige Antwort)
a) Pyrovaleron
b) Mephedron
c) Flephedron
d) Mescalin
e) Ethylon

\section{Frage 3}

Methoxetamin ist (Einfachauswahl, 1 richtige Antwort)
a) ein Halluzinogen
b) ein Wirkstoff aus der Pflanze Argyreia nervosa
c) ein Analog des Phencyclidins
d) in der Schweiz bisher nicht in Erscheinung getreten
e) ein Stimulans

\section{Frage 4}

Welche Aussage trifft nicht zu? Levamisol (Einfachauswahl, 1 richtige Antwort)
a) ist ein Anthelmintikum
b) wird dem Kokain beigesetzt
c) führt zu einer Leukozytose
d) kann zu einer Vaskulitis führen
e) wird mit dem Auftreten von Hautnekrosen in Zusammenhang gesehen

\section{Frage 5}

Kratom (Einfachauswahl, 1 richtige Antwort)
a) ist ein Analog des Ketamins
b) wird aus der Pflanze Mitragyna speciosa hergestellt 
c) ist der Stassenrufname eines der neuen synthetischen Cathinone

d) stammt aus der Pflanze Salvia divinorum

e) wurde zur Erforschung der Cannabinoid-Rezeptoren entwickelt 


\section{Autor und Korrespondenzadresse ((Ü2))}

Dr. med. Hugo Kupferschmidt

\section{Direktor}

Schweizerisches Toxikologisches Informationszentrum (STIZ)

Freiestrasse 16

8032 Zürich

hkupferschmidt@toxi.ch

\section{Bibliographie ((Ü3))}

1. Gibbons S: Legal highs. Clin Toxicol 2012; 50: 15-24.

2. Hill SL, Thomas SHL: Clinical toxicology of newer recreational drugs. Clin Toxicol 2011; 49: 705-719.

3. Rosenbaum CD, Carreio SP, Babu KM: Here today, gone tomorrow ... and back again? A review of herbal marijuana alternatives (K2, Spice), synthetic cathinones (bath salts), kratom, Salvia divinorum, methoxetamine, and piperazines. J Med Toxicol 2012; Jan 25. Epub ahead of print.

4. Prosser JM, Nelson LS: The toxicology of bath salts: A review of synthetic cathinones. J Med Toxicol 2011; Nov 23. Epub ahead of print.

5. Bruggisser M, Ceschi A, Bodmer M, Wilks MF, Kupferschmidt H, Liechti ME: Retrospective analysis of stimulant abuse cases reported to the Swiss Toxicological Information Centre during 1997-2009. Swiss Med Wkly 2010; 140: w13115.

6. Schep LJ, Slaughter RJ, Vale JA, Beasley DM, Gee P: Review: The clinical toxicology of the designer "party pills" benzylpiperazine and trifluoromethylphenylpiperazine. ClinToxicol 2011; 49: 131-141.

7. Hofer KE, Grager B, Müller DM, Rauber-Lüthy C, Kupferschmidt H, Rentsch KM, et al.: Ketamine-like effects after recreational use of methoxetamine. Ann Merg Med 2012; Jan 11. Epub ahead of print. 
DOI 10.1024/1661-8157/a000923

((PX10_Rubrik CME-Antworten))

\section{Antworten zu den Fragen zu Neuen Drogen aus PRAXIS Nr. 9}

\section{Frage 1}

Antwort d) ist richtig.

Das Auftreten einer Vaskulitis mit Hautnekrosen wurde auf die Beimischung des Anthelmintikums Levamisol zu Kokain zurückgeführt. Im Zusammenhang mit den neuen synthetischen Cathinonen wurde diese unerwünschte Wirkung bisher nicht beschrieben.

Die Symptome Tachykardie, Agitation, erhöhte Körpertemperatur und arterielle Hypertonie gehören alle zum sympathomimetischen Toxidrom.

\section{Frage 2}

Antwort d) ist richtig.

Mescalin gehört nicht zur Gruppe der Cathinone, sondern ist ein halluzinogener Wirkstoff aus dem Kaktus Lophophora williamsii.

Pyrovaleron, Mephedron, Flephedron und Ethylon sind alles Cathinone (beta-keto-Amphetamine).

\section{Frage 3}

\section{Antwort c) ist richtig.}

Methoxetamin ist wie Ketamin an Analog der Droge Phencyclidins (PCP).

Es hat keine halluzinogenen Wirkungen und ist auch kein Stimulans, sondern wirkt ähnlich wie Ketamin als dissoziatives Anästhetikum. Es wird nicht aus Pflanzen gewonnen. Einzelfälle wurden auch in der Schweiz mitgeteilt (Hofer KE, et al. Ann Merg Med 2012; Jan 11 [Epub ahead of print])..

\section{Frage 4}

Antwort c) ist richtig.

Eine unerwünschte Wirkung des Anthelmintikums Levamisol ist die Leukopenie.

Levamisol wird in den letzten Jahren dem Kokain als Streckmittel und als Wirkungsverstärker beigesetzt. Es kann auch zu einer Vaskulitis mit Hautnekrosen führen.

\section{Frage 5}

Antwort b) ist richtig.

Kratom ist eine Zubereitung aus der ostasiatischen Pflanze Mitragyna speciosa.

Es ist nicht ein Analog des Ketamins und auch kein Cathinon, sondern enthält den Wirkstoff Mitragynin, der opiatartige Effekte erzeugt. Damit spielt Mitragynin auch keine Rolle bei der Erforschung der Cannabinoid-

Rezeptoren. 